\title{
Evaluation of antibacterial activity of different mangrove plant extracts
}

\author{
P.D. Abeysinghe, R.P. Wanigatunge \\ Department of Botany, University of Ruhuna, Matara, Sri Lanka, pushpa@bot.ruh.ac.lk, \\ R.N. Pathirana \\ Department of Chemistry University of Ruhuna, Matara, Sri Lanka, ranjithnp@chem.ruh.ac.lk
}

\begin{abstract}
Antibacterial activity of mature leaves, tender leaves and bark extracts of Avicennia marina, Avicennia officinalis and Bruguiera sexangula were evaluated using Soxhelt extraction method. Petroleum ether, chloroform, ethyl acetate and ethanol were used as solvents in order to get the plant extracts. The antibacterial activity was screened by using agar diffusion technique against pathogenic bacteria species of Staphylococcus sp. (from urine), Proteus sp. (from a wound), Escherichia coli (from infected blood), Shigella sp. (from a wound) and Pseudomonas sp. (from a wound). The length of inhibition zone was measured in millimeters from the edge of the well to the edge of the inhibition zone. Twelve different plant extracts in A. marina, A. officinalis and B. sexangula exhibited different degree of growth inhibition against tested bacterial strains. Mature leaf extracts of A. marina and tender leaf extracts of $A$. officinalis in ethyl acetate exhibited promising antibacterial activity than other plant extracts. All plant extracts in ethyl acetate showed strong inhibition compared to other extracts on all tested bacterial strains. Among all bacterial strains, Pseudomonas sp. and E. coli showed considerable growth inhibition against almost all plant extracts. Mature leaf extract of only A. marina was used for further investigations since a large amount of tender leaves of $A$. officinalis was not readily available. Charcoal treated mature leaf extracts of A. marina showed more inhibition for all tested bacterial strains than untreated plant extracts. Combinations of mature leaf extracts of A. marina in different solvents failed to exhibit synergistic activity against tested bacterial strains. Most combinations showed antagonistic effect against Proteus sp., E. coli and Shigella sp. Antibacterial activity of plant extracts of A. marina gradually declined during 4 months after extraction, for all tested bacterial strains. Components of mature leaf extracts of A. marina in chloroform, ethyl acetate and ethanol were separated by Analytical Thin Layer Chromatography (ATLC) when the eluents were hexane and diethyl ether. Isolated components in Preparative Thin Layer Chromatography (PTLC) exhibited moderate antibacterial activity against Pseudomonas sp., Shigella sp. and E. coli. Separated components did not show any antibacterial activity against Proteus sp. and Staphylococcus sp. Phytochemical screening revealed that mature leaf of A. marina contained alkaloids, steroids, triterpenoids and flavonoids.
\end{abstract}

Key words: A. marina, antibacterial activity, plant extracts, growth inhibition

\section{Introduction}

Microorganisms have potential to cause human diseases. Most of the time viruses, bacteria and fungi act as major pathogenic organisms. The discovery of antibiotics in the early twentieth centaury provided an increasingly important tool to combat bacterial diseases. As antibiotics are increasingly used and misused, the bacterial 
strains become resistant to antibiotics rapidly. Therefore, screening of antibacterial activity of medicinal plants is very important since vast number of medicinal plants have been used for centuries as remedies for human diseases. Among them extracts from different parts of mangroves and mangrove associates are widely used throughout the world. For instance, stem of Avicennia marina is used for ulcers and bark of Bruguiera sexangula is used for antitumors (Bandaranayake 1998). Mangrove and mangrove associates contain biologically active antiviral, antibacterial and antifungal compounds (Bandaranayake 1998). They provide a rich source of steroids, triterpenes, saponins, flavonoids, alkaloids and tannins (Bandaranayake 1995). Therefore, it is worth to screen mangrove plants for the presence of new antibacterial compounds to combat the normal pathogenic bacterial strains and hospital acquired antibiotic resistant bacterial strains.

The main objectives of this study were to screen antibacterial activity of some selected mangrove plant species against pathogenic and antibiotic resistant bacterial strains and also to isolate and characterization of chemical components which are responsible for antibacterial activity.

\section{Materials and Methods}

\subsection{Plant materials and preparation of plant extracts}

Mature leaves, tender leaves and bark of Avicennia marina, Avicennia officinalis and Bruguiera sexangula were used as plant materials. Sequential Soxhlet extraction method was carried out to obtain plant extracts. Fifty grams of mature leaves of each selected mangrove species were ground and extracted separately with petroleum ether $(300 \mathrm{ml})$ followed by chloroform $(300 \mathrm{ml})$, ethyl acetate $(300 \mathrm{ml})$ and finally with ethanol $(300 \mathrm{ml})$. The extraction time period was three hours for each solvent. The same procedure was followed for tender leaves and bark of tested plants. Extracts were concentrated to $3 \mathrm{ml}$ portions using a rotary evaporator at $50^{\circ} \mathrm{C}$ and stored at $4^{\circ} \mathrm{C}$.

Four mature leaf extracts of $A$. marina obtained from Soxhlet extraction method were treated with appropriate amount of activated charcoal and incubated at $40^{\circ} \mathrm{C}$ for 10 minutes in a water bath. Charcoal treated extracts were centrifuged at $10,000 \mathrm{rpm}$ for 2 minutes and the organic layer was obtained. Mature leaf extracts of A. marina were mixed with the following combinations of solvents: petroleum ether and chloroform, petroleum ether and ethyl acetate, petroleum ether and ethanol were used to test synergistic activity of plant extracts.

Plant extracts for phytochemical screening were prepared using $100 \mathrm{~g}$ of ground fresh mature leaves of $A$. marina. They were extracted using $300 \mathrm{ml}$ of $95 \%(\mathrm{v} / \mathrm{v})$ ethanol in Soxhlet apparatus. The mixture was refluxed on a steam bath about one hour, then cooled to room temperature and plant extract was washed with $50 \mathrm{ml}$ of fresh $95 \%(\mathrm{v} / \mathrm{v})$ ethanol. The final volume of the extract was measured and portions of this extracts were used in phytochemical screening for alkaloids, flavonoids, saponins, terpenoids, steroids and cardiac glycosides. 
Table 1 Tested bacterial strains for antibacterial activity.

\begin{tabular}{|l|l|}
\hline Bacterial strain & Source \\
\hline Escherichia coli (from blood) & General hospital, (Matara) \\
Proteus sp.* (from a wound) & General hospital, (Matara) \\
Pseudomonas sp. (from a wound) & General hospital, (Matara) \\
Shigella sp. (from a wound) & Faculty of medicine, (University of Ruhuna) \\
Staphylococcus sp. ${ }^{*}$ (from urine) & General hospital, (Matara) \\
\hline
\end{tabular}

*antibiotic resistant bacterial strains.

\subsection{Tested bacterial strains}

Staphylococcus sp. is resistant to many common antibiotics in use such as Ceftazidime, Cephalexin, Cotrimoxazole, Cloxacillin, Gentamycin, Kenamycin and Ticarcillin/Clavulanic acid although Proteus sp. is resistant only to Gentamycin and Kenamycin as indicated in the Microbiology report of the General Hospital, Matara 2003.

\subsection{Determination of antibacterial activity}

Antibacterial activity of tested bacterial strains was assayed using all plant extracts by agar diffusion technique (de Castillo et al. 1998). Antibacterial activity was observed as inhibition zone on Petri plates containing nutrient agar. Size of the inhibition zone was measured in millimeters using a metric ruler from the edge of the well to the edge of the inhibition zone. Petroleum ether, chloroform, ethyl acetate and ethanol were used as controls instead of plant extracts. Stability of antibacterial activity of plant extracts was tested during a period of 4 months and results were obtained once a month using agar diffusion technique. Antibacterial activity against tested bacterial strains (Table 1) was tested for components separated by PTLC.

\subsection{Separation of active components}

Mature leaf extract obtained from Soxhlet extraction was separated using Thin Layer Chromatography (TLC). Pure solvents of hexane, diethyl ether, petroleum ether, ethyl acetate and water, a mixture of hexane : diethyl ether; petroleum ether: ethyl acetate; ethyl acetate: water; hexane: water were used as mobile phase with different ratios as $9: 1,8: 2,7: 3,6: 4,5: 5,4: 6,3: 7,2: 8$ and $1: 9$. Plates were observed under UV light and developed using $\mathrm{I}_{2}$ vapour.

\subsection{Phytochemical screening}

Phytochemical screening was carried out for crude extract of mature leaves of $A$. marina for alkaloids, flavonoids, saponins, terpenoids, steroids and cardiac glycosides. Mayer's reagent and Wagner's reagent were used to detect alkaloids. Dragendorff's reagent was used to estimate the probable number of alkaloids present. Mayer's reagent was also used to determine quaternary alkaloids. Saponins were screened using froth test. Liebermann - Burchardt test and Salkoweski test were carried out to determine steroids and triterpenoids. Keller- killiani test was performed for screening cardiac glycosides. Flavonoids were tested using octanol (Harbone 1984). 


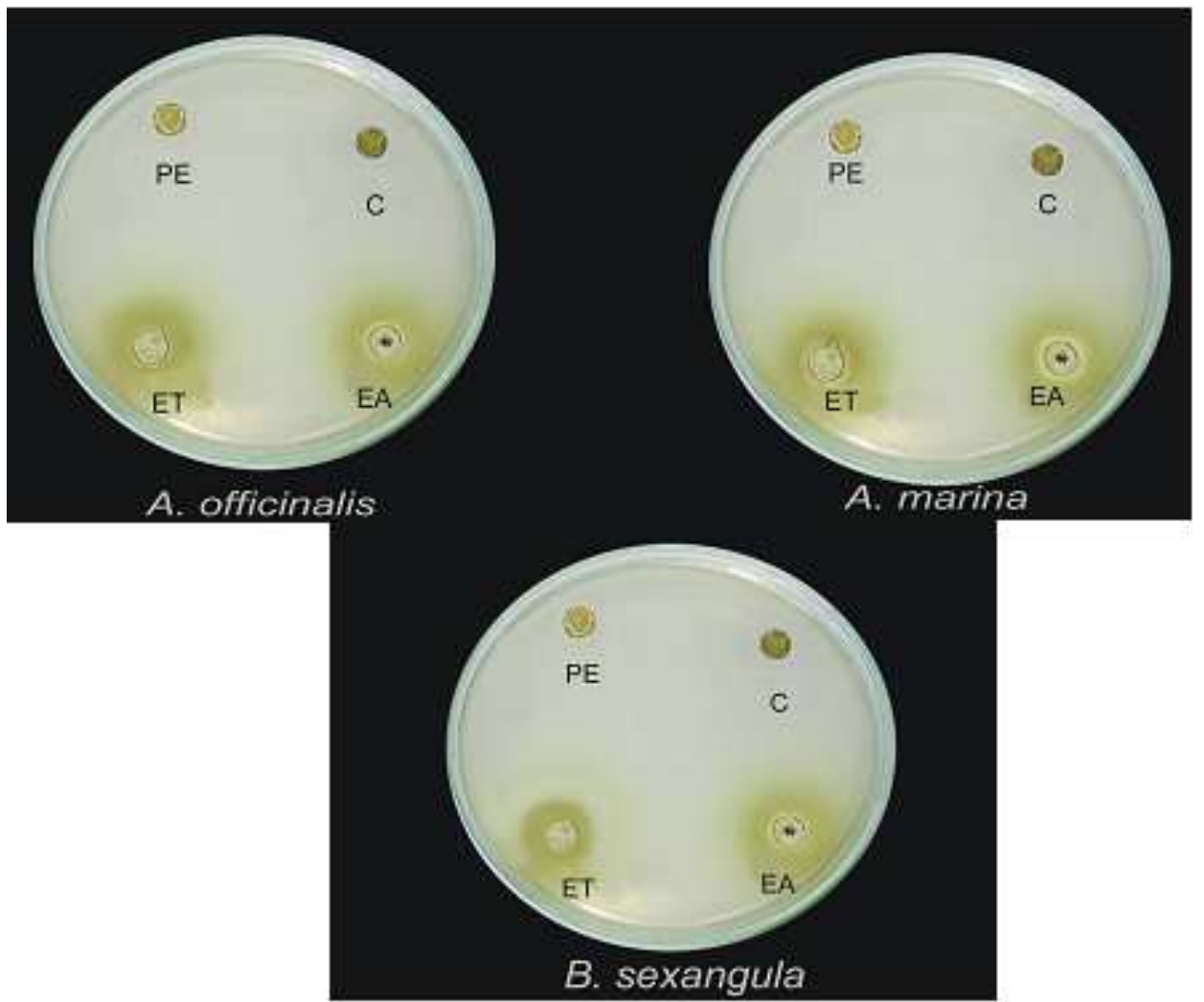

Figure 1 Inhibition of $E$. coli by plant extracts of mature leaf of $A$. marina, $A$. officinalis and $B$. sexangula extracted in petroleum ether (PE), chloroform (C), ethyl acetate (EA), and ethanol (ET) using Soxhlet extraction method.

\section{Results}

Twelve different extracts from A. marina, A. officinalis and B. sexangula exhibited different degree of growth inhibition against tested bacterial strains (Table 2). According to Table 2, mature leaves of $A$. marina and tender leaves of $A$. officinalis exhibited considerable antibacterial activity against tested bacterial strains. Further tests were carried out using only mature leaves of $A$. marina because tender leaves of $A$. officinalis are not easily obtainable.

Plant extracts of mature leaf of $A$. marina showed more inhibition than mature leaf extracts of $A$. officinalis and B. sexangula against E. coli, Staphylococcus sp., and Shigella sp. (Fig. 1 and Table 2). Tender leaf extracts of A. officinalis exhibited more pronounced inhibition against Staphylococcus sp. and Pseudomonas sp. (Fig. 2 and Table 2). Bark extracts of A. marina showed strong inhibition against Shigella sp. and E. coli (Fig. 3 and Table 2) compared to the extracts of B. sexangula and A. officinalis.

Mature leaf extract of $A$. officinalis was unable to inhibit the growth of Shigella sp. and Staphylococcus sp. while tender leaf extract did not exhibit antibacterial activity against Proteus sp. Moreover, none of the tender leaf extracts of B. sexangula were able to inhibit Proteus sp., Pseudomonas sp. and Shigella sp. Also mature 

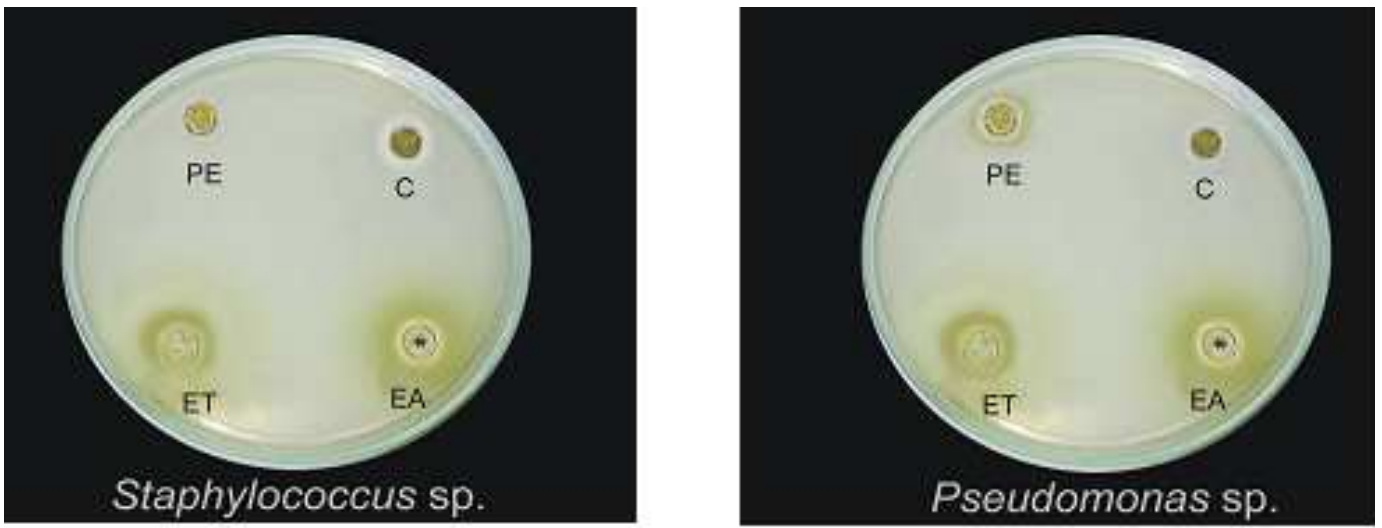

Figure 2 Inhibition of Staphylococcus sp. and Pseudomonas sp. by plant extracts of tender leaf of $A$. officinalis extracted in petroleum ether (PE), chloroform (C), ethyl acetate (EA) and ethanol (ET) using Soxhlet extraction method.
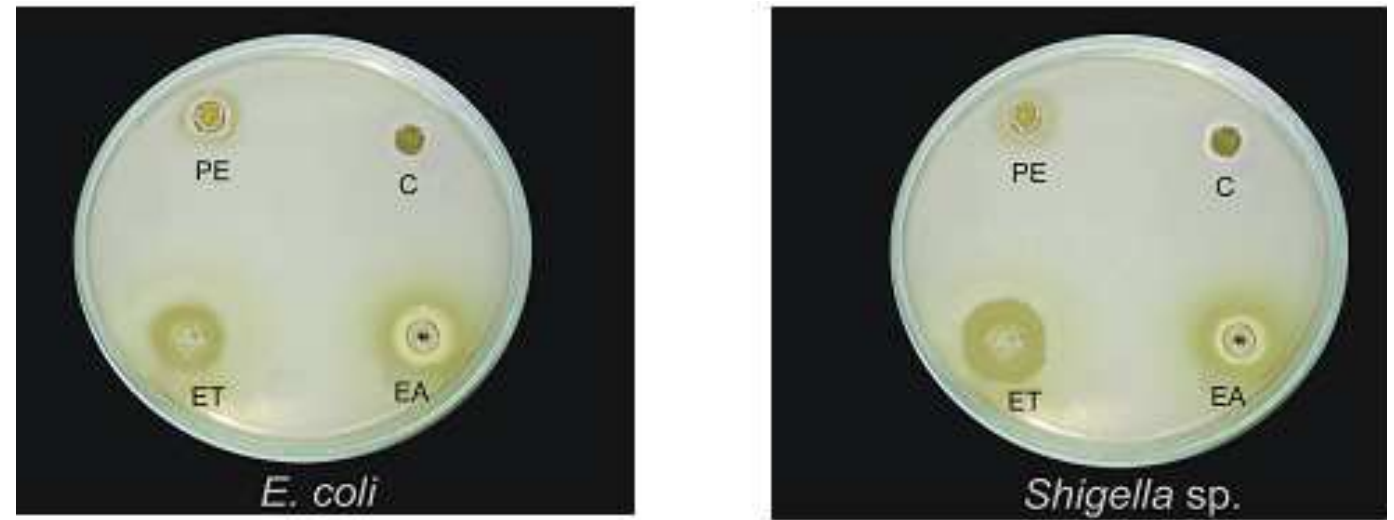

Figure 3 Inhibition of $E$. coli and Shigella sp. by plant extracts of bark of $A$.marina extracted in petroleum ether (PE), chloroform (C), ethyl acetate (EA) and ethanol (ET) using Soxhlet extraction method.

leaf extract of $B$. sexangula did not exhibit antibacterial activity against Pseudomonas sp. and bark extracts were unable to inhibit Staphylococcus sp (Table 2).

No inhibition of the growth of Proteus sp., Staphylococcus sp. and Shigella sp. was observed in extracts in petroleum ether except bark extract of A. marina. Ethyl acetate extracts of bark of $A$. officinalis, mature leaf and tender leaf extract of $A$. marina and chloroform extract of mature leaf of $A$. marina were able to inhibit the growth of all tested bacterial strains. Ethanolic extract of bark of $A$. officinalis greatly inhibited, the growth of Pseudomonas sp, E. coli and Shigella sp. Controls did not exhibit inhibitory effect against any of the tested bacterial strains. Charcoal treated mature leaf extracts of A. marina were able to inhibit the growth of all tested bacterial strains more than untreated extracts (Table 3). None of the tested possible combinations of mature leaf extracts were able to exhibit synergistic activity 


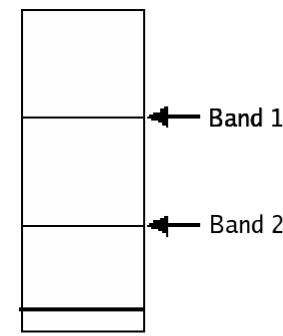

I Mature leaves in chloroform

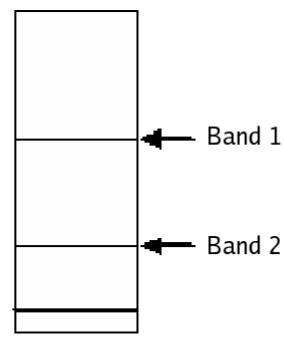

II Mature leaves in ethanol

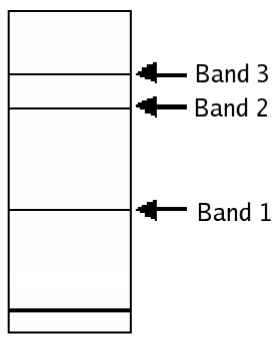

III Mature leaves in ethyl acetate

Figure 4 A diagrammatic representation of PTLC plates showing separated bands in plant extracts of mature leaves in chloroform, ethanol and ethyl acetate when the eluents were hexane: diethyl ether 4: 6 .

Table 2 A comparison of the degree of growth inhibition of bacterial species in millimeters ( $\mathrm{mm}$ ) from the edge of the well to the edge of the inhibition zone by charcoal treated $(T)$ and untreated (UT) plant extracts of mature leaves of $A$. marina in petroleum ether (PE), chloroform (C), ethyl acetate (EA) and ethanol (ET).

\begin{tabular}{|c|c|c|c|c|c|c|c|c|c|c|c|c|}
\hline \multirow{2}{*}{\multicolumn{3}{|c|}{ Plant material }} & \multicolumn{2}{|c|}{ Proteus sp. } & \multicolumn{2}{|c|}{ Staphylococcs sp. } & \multicolumn{2}{|c|}{ Pseudomonas sp. } & \multicolumn{2}{|c|}{ E. coli } & \multicolumn{2}{|c|}{ Shigella sp. } \\
\hline & & & $\overline{\mathrm{UT}}$ & $\mathrm{T}$ & UT & $\mathrm{T}$ & $\overline{\mathrm{UT}}$ & $\mathrm{T}$ & $\overline{\mathrm{UT}}$ & $\overline{\mathrm{T}}$ & $\mathrm{UT}$ & $\mathrm{T}$ \\
\hline \multirow{4}{*}{ 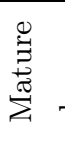 } & & $\mathrm{PE}$ & - & - & - & - & 2 & 3 & 1 & 2 & - & - \\
\hline & & $\mathrm{C}$ & 1 & 3 & 1 & 1 & 1 & 3 & 2 & 2 & 2 & 3 \\
\hline & & EA & 4 & 5 & 4 & 7 & 4 & 6 & 6 & 8 & 7 & 9 \\
\hline & & $\mathrm{ET}$ & 2 & 4 & - & - & - & - & 5 & 7 & 5 & 8 \\
\hline
\end{tabular}

( - mark indicates no inhibition)

against tested bacterial strains. Most combinations exhibited antagonistic effect against Proteus sp., E. coli and Shigella sp. (Table 4). The degree of antibacterial activity of mature leaf extract of $A$. marina decreased with time for all tested bacterial strains. When TLC was carried out, chloroform and ethanol extracts of mature leaves separated into two bands and ethyl acetate extract of mature leaves separated into three bands (Fig. 4). Separated components of TLC could inhibit the growth of tested bacterial species except Proteus sp. and Staphylococcus sp. (Table 5). According to phytochemical screening, mature leaves of A. marina contained alkaloids, steroids and flavonoids.

\section{Discussion}

A. marina, A. officinalis and B. sexangula were used as test plants due to the presence of much evidence that prove their therapeutic value against microbial infections (Bandaranayake 1998). Also preliminary studies have been demonstrated that the mangrove plant extracts have antibacterial activity against pathogenic bacterial strains; Staphylococcus sp. and E. coli and Pseudomonas sp. (Abeysinghe et al. 2002) and antibiotic resistant bacterial strains; Staphylococcus sp and Proteus sp. (Abeysinghe et al. 2003). The results of the present study clearly showed that mangrove plant extracts showed antibacterial activity against tested pathogenic bacterial strains including antibiotic resistant strains. The effectiveness of the active 
Table 3 Degree of growth inhibition of bacterial species measured in millimeters ( $\mathrm{mm}$ ) from the edge of the well to the edge of the inhibition zone by possible combinations of mature leaf extracts of $A$. marina in petroleum ether (PE), chloroform, ethyl acetate (EA) and ethanol (ET)

\begin{tabular}{|c|c|c|c|c|c|c|c|c|c|c|c|c|c|c|c|}
\hline \multirow[b]{2}{*}{ Bacterial strains } & \multicolumn{15}{|c|}{ Combination of plant extracts } \\
\hline & $\frac{1}{2}$ & $\circlearrowright$ & $\underset{4}{\overleftrightarrow{4}}$ & 年 & $\begin{array}{l}0 \\
+ \\
\text { II }\end{array}$ & 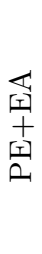 & $\begin{array}{l}E \\
\text { 季 } \\
+ \\
\text { II } \\
0\end{array}$ & $\begin{array}{l}\underset{I}{1} \\
+ \\
0\end{array}$ & $\begin{array}{l}E-1 \\
+ \\
+\end{array}$ & $\begin{array}{l}E \\
\underset{1}{+1} \\
+ \\
+\end{array}$ & $\begin{array}{l}\underset{1}{1} \\
+ \\
0 \\
+ \\
+1 \\
0\end{array}$ & $\begin{array}{l}-1 \\
\text { I } \\
+ \\
0 \\
+ \\
+ \\
0 \\
\end{array}$ & 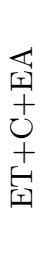 & 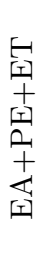 & 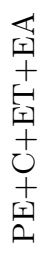 \\
\hline Proteus sp. & - & 1 & 3 & 2 & 1 & 2 & 1 & 2 & 1 & 3 & 2 & 2 & 4 & - & - \\
\hline Pseudomonas sp. & 1 & 1 & 7 & - & - & - & - & 4 & - & - & - & - & - & - & - \\
\hline Staphylococcus sp. & - & 1 & 5 & - & 1 & - & - & - & - & - & - & - & - & - & - \\
\hline Shigella sp. & - & 2 & 8 & 4 & 1 & 2 & 4 & 9 & 4 & 6 & 4 & 2 & 4 & 5 & 7 \\
\hline E. coli & 1 & 1 & 8 & 6 & 2 & 5 & - & 7 & - & 6 & 3 & 1 & 4 & 3 & 4 \\
\hline
\end{tabular}

( - indicates no inhibition).

Table 4 Level of inhibition caused by the components of mature leaf extracts of $A$. marina in chloroform (MC), in ethanol ((ME) and in ethyl acetate (MEA) separated by PTLC measured in millimeters $(\mathrm{mm})$ from the edge of well to the edge of inhibition zone against Proteus sp. (Pr), Staphylococcus sp. (St), Pseudomonas sp. (Ps), E. coli and Shigella sp. (Sh) by TLC method,B1- band one, B2- band two, B3-band three )

\begin{tabular}{|l|l|l|l|l|l|l|l|}
\hline \multirow{2}{*}{ Bacterial strain } & \multicolumn{7}{|c|}{ Isolated bands } \\
\cline { 2 - 8 } & MCB 1 & MCB 2 & MEB 1 & MEB 2 & MEAB 1 & MEAB 2 & MEAB 3 \\
\hline Pr & - & - & - & - & - & - & - \\
Ps & - & 2 & 1 & 1 & - & - & - \\
St & - & - & - & - & - & - & - \\
E.coli & 2 & 1 & 1 & - & 1 & 2 & - \\
Sh & 1 & 1 & 2 & - & - & - & - \\
\hline
\end{tabular}

( -mark indicates no inhibition).

compounds present in plant extracts cause the production of growth inhibition zones that appear as clear areas surrounding the wells. Antibacterial activity may be due to active components which are present in plant extracts. However, some plant extracts were unable to exhibit antibacterial activity against tested bacterial strains. These bacterial strains may have some kind of resistance mechanisms e.g. enzymatic inactivation, target sites modification and decrease intracellular drug accumulation (Schwarz and Noble 1999) or the concentration of the compound used may not be sufficient. No inhibition was observed with controls, which proves that solvents could not act as antibacterial agents. Further investigations were done using extracts of mature leaves of $A$. marina only, since they showed considerable antibacterial activity. In almost all tests, crude ethyl acetate extracts showed better inhibition against all tested bacterial strains, indicating that active ingredients in plant materials could be extracted into ethyl acetate. However, highest antibacterial activity was observed against E. coli. 
Charcoal treated plant extracts showed more inhibition against all tested bacterial strains than untreated plant extracts. It may be assumed that plant pigments may increase the survival ability of bacteria. The degree of antibacterial activity of plant extracts decreased during the period of four months after extraction. It may be due to degradation or volatility of antibacterial compounds or they may have converted into non-antibacterial compounds. A combination of plant extracts in different solvents unable to exhibit synergistic activity against tested bacterial strains. Mixing of plant extracts in different solvents seems to have diluted down the toxic effect against tested bacterial stains or might have increased the survival ability of pathogenic bacteria. TLC was carried out using different solvent systems. But only hexane and diethyl ether solvent system gave best separation. Separated components showed positive results against Pseudomonas sp., E. coli and Shigella sp. whereas antibacterial activity was not observed against Staphylococcus sp and Proteus sp. According to preliminary studies, it has been reported that active components from mature leaves of A. marina have been separated using TLC (Withanawasm 2002).

Phytochemical screening of mature leaf extracts of $A$. marina revealed the presence of biologically active substances such as alkaloids, steroids, triterpenoids and flavonoids. The results obtained from preliminary phytochemical screening are comparable with the results reported earlier (Bandaranayake, 1995). Preliminary phytochemical screening of Luminetzera racemosa reveled the presence of secondary metabolites such as alkaloids, flavonoids and steroids (Abeysinghe et al., 2003). These secondary metabolites may exert antibacterial activity against tested bacterial strains. In addition to above tested phytochemical groups tannins, anthocyanins, polyphenols, coumarins and essential oils should be subjected to phytochemical screening. It is promising that the tested mangrove plant species could be used to synthesis novel antibiotics for bacterial infections, especially for antibiotic resistant bacterial infections. Further research is necessary for successful separation, purification and characterization of biologically active compounds using chromatographic methods and spectroscopic techniques. Further studies are being carried out in order to separate the individual components that are present in plant extracts of $A$. marina using column chromatography.

\section{References}

Abeysinghe PD, Withanawasam M, Pathirana RN, Abeysinghe S. 2002. Preliminary in vitro screening of antibacterial compounds of some mangrove plant extracts for clinical isolates from different sources. Proceeding of the First Science Symposium, University of Ruhuna, 22-25 pp.

Abeysinghe PD, Wijesekara D, Pathirana RN. 2003. Inhibition of growth of antibiotic resistant Staphylococcus sp. and Proteus sp. by mangrove plant extracts. Proceeding of the First Science Symposium, University of Ruhuna, 1-9 pp.

Bandaranayake WM. 1995. Survey of mangrove plants from Northern Australia for phytochemical constituents and uv-absorbing compounds. Current Topics in Phytochemistry (Life Science Advances) 14:69-78 
Bandaranayake WM. 1998. Traditional and medicinal uses of mangroves. Mangroves and Salt Marshes. 2:133-148.

de Castillo MC, de Nader OM, de R. Holgado AP. 1998. In vitro comparison of disk diffusion and agar dilution antibiotic susceptibility test methods for Neisseria gonorrhoeae. 93(4):517.

Harbone JB. 1984. Phytochemical methods. 2nd edition. Chapman and Hall, London.

Schwarz S, Noble WC. 1999. Aspects of bacterial resistance to antimicrobials used in veterinary dermatological practice. 163-176 pp.

Withanawasam DM. 2002. Preliminary in vitro screening of antibacterial and antifungal compounds of mangrove plant extracts for pathogens from different sources.

\section{Acknowledgments}

This work is partially funded by the TWAS (Third World Academy of Sciences) award to the first author. 